\title{
Feline Parvovirus Seroprevalence Is High in Domestic Cats from Disease Outbreak and Non-Outbreak Regions in Australia
}

\author{
Elizabeth Jenkins ${ }^{1}$, Conor Davis ${ }^{1}$, Maura Carrai ${ }^{1}$, Michael P. Ward ${ }^{1}{ }^{\circledR}$, Susan O'Keeffe ${ }^{2}$, \\ Martine van Boeijen ${ }^{3}$, Louise Beveridge ${ }^{4}$, Costantina Desario ${ }^{5}$, Canio Buonavoglia ${ }^{5}$, \\ Julia A. Beatty ${ }^{1}\left(\mathbb{D}\right.$, Nicola Decaro ${ }^{5}$ (D) and Vanessa R. Barrs ${ }^{1, *(D)}$ \\ 1 Sydney School of Veterinary Science, Faculty of Science, University of Sydney, Camperdown 2050, Australia; \\ elizabeth.jenkins@sydney.edu.au (E.J.); cdav6397@uni.sydney.edu.au (C.D.); \\ maura.carrai@sydney.edu.au (M.C.); michael.ward@sydney.edu.au (M.P.W.); \\ julia.beatty@sydney.edu.au (J.A.B.) \\ 2 School of Veterinary and Life Sciences, Murdoch University, Murdoch 6150, Australia; \\ S.O'Keeffe@murdoch.edu.au \\ 3 Perth Cat Hospital, West Leederville 6007, Australia; drmartine@perthcat.vet \\ 4 Bedford-Dianella Vet Centre, Bedford 6052, Australia; louise_bedfordvet@hotmail.com \\ 5 Department of Veterinary Medicine, University of Bari, Valenzano, 70121 Bari, Italy; \\ costantina.desario@uniba.it (C.D.); canio.buonavoglia@uniba.it (C.B.); nicola.decaro@uniba.it (N.D.) \\ * Correspondence: vanessa.barrs@sydney.edu.au; Tel.: +61-2-9351-3437
}

Received: 14 January 2020; Accepted: 12 March 2020; Published: 16 March 2020

check for updates

\begin{abstract}
Multiple, epizootic outbreaks of feline panleukopenia (FPL) caused by feline parvovirus (FPV) occurred in eastern Australia between 2014 and 2018. Most affected cats were unvaccinated. We hypothesised that low population immunity was a major driver of re-emergent FPL. The aim of this study was to (i) determine the prevalence and predictors of seroprotective titres to FPV among shelter-housed and owned cats, and (ii) compare the prevalence of seroprotection between a region affected and unaffected by FPL outbreaks. FPV antibodies were detected by haemagglutination inhibition assay on sera from 523 cats and titres $\geq 1: 40$ were considered protective. Socioeconomic indices based on postcode and census data were included in the risk factor analysis. The prevalence of protective FPV antibody titres was high overall (94.3\%), even though only $42 \%$ of cats were known to be vaccinated, and was not significantly different between outbreak and non-outbreak regions. On multivariable logistic regression analysis vaccinated cats were 29.94 times more likely to have protective FPV titres than cats not known to be vaccinated. Cats from postcodes of relatively less socioeconomic disadvantage were 5.93 times more likely to have protective FPV titres. The predictors identified for FPV seroprotective titres indicate targeted vaccination strategies in regions of socioeconomic disadvantage would be beneficial to increase population immunity. The critical level of vaccine coverage required to halt FPV transmission and prevent FPL outbreaks should be determined.
\end{abstract}

Keywords: Carnivore protoparvovirus; feline parvovirus; feline panleukopenia; haemagglutination inhibition; seroprevalence

\section{Introduction}

Feline panleukopenia (FPL), caused by Carnivore protoparvovirus 1 (Order Ortervirales, Family Parvoviridae, Subfamily Parvovirinae), is characterised by severe enteritis and immunosuppression and has high morbidity and mortality. Feline parvovirus (FPV) causes $95 \%$ of cases, with the remaining $5 \%$ 
caused by canine parvovirus (CPV), including the antigenic variants CPV-2a, $-2 b$, and $2 c$, also known as “CPV-2a-like" viruses [1].

In Australia FPL has been encountered only sporadically since the mid-1970s when the use of effective vaccines became widespread. Between 2014 and 2018, FPL re-emerged in eastern Australia, causing multiple epizootic outbreaks with high mortality predominantly among shelter-housed cats [2]. FPV was identified as the causative agent of the first outbreak in 2014 in the state of Victoria [2]. Outbreaks in New South Wales (NSW) first occurred in 2016 and were caused by a different and distinctive FPV strain, ruling out geographic spread from Victoria. Most affected cats were unvaccinated, or vaccination history was unknown; hence low population immunity was hypothesised to be a major driver of FPL re-emergence [1,2].

Serum antibody titres to FPV, determined using virus neutralisation (VN) or haemagglutination inhibition (HI) assays or ELISAs (laboratory-based or point-of-care), are used to predict protection from disease [3-6]. A cut-off $\geq 1: 40$ is used widely to define the minimum HI titre that confers serological protection, but a range of cut-offs from 0 to $\geq 1: 80$ have been reported [3,4,7-9].

A single previous study of the seroprevalence of FPV antibodies in Australian cats (Felis catus) undertaken in 1981 and involving 92 unowned, free-living cats in south-eastern Australia, found a seroprevalence of 79\%, suggesting widespread exposure to FPV [10]. FPV seroprevalence data among shelter-housed cats or owned cats in Australia have not been reported previously. Factors associated with FPV seropositivity in other regions include previous vaccination [11], increasing age, being owned, and being desexed $[8,9,12,13]$.

The aim of this study was to determine (i) FPV seroprevalence and predictors of seropositivity among shelter-housed and owned cats, and (ii) whether seroprevalence differed in these two cohorts between an outbreak location in eastern Australia (Sydney) and a region with no recent history of FPL outbreaks in western Australia (Perth).

\section{Materials and Methods}

\subsection{Study Populations}

Blood samples were collected from four groups of cats: (i) owned and (ii) shelter-housed cats from Sydney, NSW, a region in which recurrent FPL season outbreaks have been occurring since December 2016 [2]; and (iii) owned and (iv) shelter-housed cats from Perth, Western Australia (WA), where no FPL outbreaks have been reported in recent decades. Blood samples were collected from a single large shelter in Perth and from three smaller shelters in Sydney. Blood samples were collected from owned cats presenting for veterinary consultations collected from seven veterinary hospitals in Sydney and from two veterinary hospitals in Perth. In Sydney, four of the veterinary hospitals included were in close proximity to the previously reported outbreaks and were participating in a subsidised vaccination and desexing scheme offered by a charitable feline organisation. A power analysis was performed to determine the number of cats to be sampled. Based on an estimated minimum seroprevalence of $15 \%$ to $40 \%$ among shelter-housed cats, and 50\% to $70 \%$ among owned cats, the minimum total sample size required to detect a difference in seroprevalence between these groups, with confidence set at $95 \%$, significance of $p<0.05$, and power $\geq 80 \%$, was 200 cats overall [14]. The sample size required for risk factor analysis an odds ratio of 1.5 to be detected with $95 \%$ confidence and $40 \%$ precision assuming a $20 \%$ prevalence of the risk factor in cats with non-protective antibody titres, and a minimum ratio of cats with protective titres to cats with non-protective titres of 1.5 was 142 cats with non-protective titres [15], which would be achieved by sampling 400 cats overall (100 in each group).

\subsection{Sampling and Data Collection}

Whole blood (1-3 mL) was collected via jugular venepuncture and serum was separated and stored frozen at $-20{ }^{\circ} \mathrm{C}$ for up to 4 weeks, then transferred for storage at $-80{ }^{\circ} \mathrm{C}$ until batch testing. 
Prospective sampling was performed from June 2018 to July 2019. Additionally, 35 stored sera collected from July to August 2015, from owned cats in Perth (non-outbreak region) were included.

Data collected for each cat sampled included age, sex, desexing status, breed, location (post code), reason for presentation (health check, injured or unwell, vaccination, desexing, stray, other), time in possession of owner or shelter, other dogs or cats in the household (yes or no), health status (healthy, defined as fit to vaccinate, sick or injured), current medications, feline leukemia virus (FeLV) antigen status, feline immunodeficiency virus (FIV) antibody status, FPV vaccination status, time since last vaccination ( $<6$ months ago, 6-12 months ago, 1-3 years ago, $>3$ years ago and type of vaccination (attenuated or inactivated). The study was approved by the University of Sydney Animal Care and Ethics Committee, Approval No. 2017/1218.

\subsection{Haemagglutination Inhibition (HI) Serology}

FPV antibody titres were determined by HI assays using sera after complement inactivation, as previously described, with minor modifications [16,17]. Briefly, serum was diluted in phosphate-buffered saline (PBS) $\mathrm{pH} 5.6$ and mixed with an equal amount $\mathrm{v} / \mathrm{v}$ of undiluted porcine red blood cells (RBC), then centrifuged after overnight incubation at $4{ }^{\circ} \mathrm{C}$, at $800 \times g$ for 5 min at $4{ }^{\circ} \mathrm{C}$ and the supernatant was harvested. HI assays were performed in 96-well V-bottom plates (Nuova Aptaca, Canelli-AT, Italy) using $25 \mu \mathrm{L}$ of diluted sera mixed with $25 \mu \mathrm{L}$ of an FPV suspension containing 10 haemagglutinating units (FPV field strain 20/05), and doubling dilutions from 1:10 to 1:2560. Plates were incubated at room temperature for $1 \mathrm{~h}$, then at $4{ }^{\circ} \mathrm{C}$ for $30 \mathrm{~min}$, after which $50 \mu \mathrm{L}$ of a solution containing $0.1 \%$ porcine RBC suspension and $2 \%$ foetal bovine serum (Mediatech, Inc., Manassas, VA, USA) in PBS pH 7.2 were added to each well. Plates were read after overnight incubation at $4{ }^{\circ} \mathrm{C}$. A titre of $\geq 1: 40$ was considered protective.

\subsection{Statistical Analysis}

Descriptive analysis was performed by creating frequency distributions for categorical variables and estimating medians for continuous variables. Statistical analyses were performed using the software package IBM SPSS Statistics v24. For univariable analysis of the association between FPV protection status and categorical predictor variables, Chi-squared tests of association were used, with a $p$-value set at 0.05 for statistical significance and a protective titre was defined as an $\mathrm{HI}$ titre $\geq 1: 40[4,18]$. Seroprevalence confidence intervals (95\%) were calculated using the Clopper-Pearson exact test.

Socioeconomic data were sourced from the Australian Bureau of Statistics Socio-Economic Indexes for Areas (SEIFA) data cube from 2011 based on postcode and published by the Australian Bureau of Statistics in 2013, and the index of relative socio-economic disadvantage (IRSD), the index of relative socio-economic advantage and disadvantage (IRSAD), the index of economic resources (IER), and the index of education and occupation (IEO) [19] were extracted. Index values for each cat included in the study were based on its reported postcode. The association between each of the four indices and FPV protection status (protective vs. non-protective titre) was assessed using Mann-Whitney U tests, with a $p$-value of 0.05 used for statistical significance.

A multivariable logistic regression model was fit to the data using predictors significantly $(p<0.05)$ associated with FPV protection status analysis. A separate logistic regression analysis was performed using SEFIA variables included as categorical variables (above median vs. median or below median). The association between FPV titre and vaccination status was assessed with Kruskal-Wallis one-way analysis of variance, with a $p$-value of 0.05 used for statistical significance.

\section{Results}

\subsection{Animals}

A total of 523 cats were included in the study comprising 282 cats from Sydney (outbreak-region) and 241 cats from Perth (non-outbreak region). The geographic origins of cats sampled in the study are 
shown in Figure 1. Of the 523 cats, 234 were shelter-housed and 289 were owned. The median age of cats overall was 1.75 years (range: 7 weeks to 21 years). The median age of cats within each group was 1 year and 1.1 years for Sydney owned and shelter-housed cats, respectively and 10 years and 1 year for Perth owned and shelter-housed cats, respectively. The median age of cats overall from Sydney and Perth was 1 year and 3.5 years, respectively.

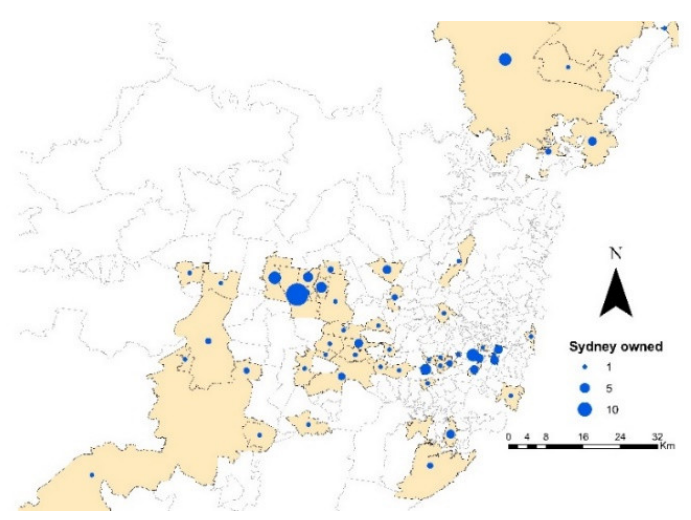

(a)

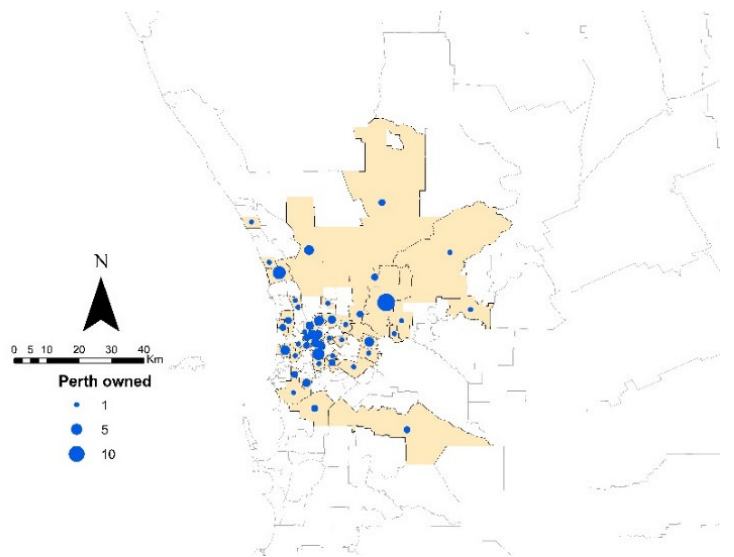

(c)

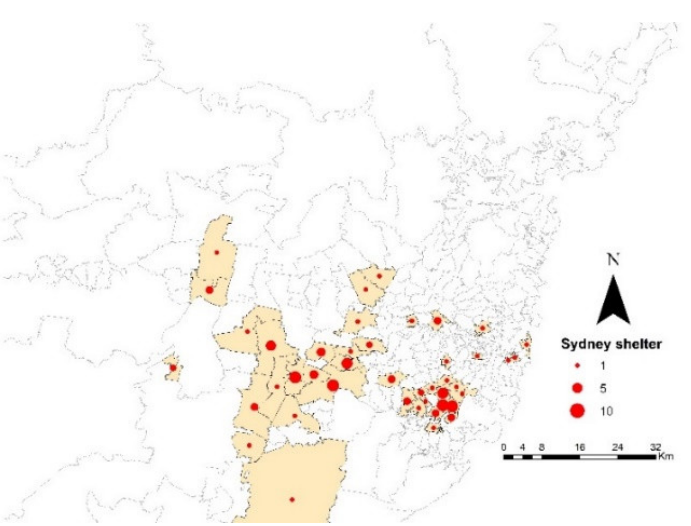

(b)

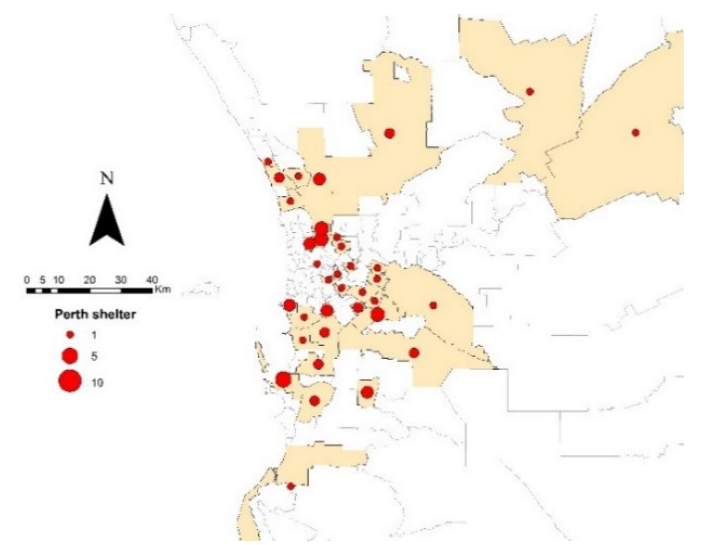

(d)

Figure 1. Geographic origin of samples collected in this study. Samples collected from shelter-housed cats are represented by blue dots, and samples from owned cats are represented by red dots. The size of the dots is proportional to the number of samples collected from one postcode area. The postcode areas sampled are shaded in beige. Owned (a) and shelter-housed (b) cats in the outbreak region (Greater Sydney); owned (c) and shelter-housed (d) cats in the non-outbreak region (Greater Perth).

Of cats from Sydney, $29 \%$ of owned and $47 \%$ of shelter-housed cats were vaccinated; of cats from Perth, 19\% and 78\% of owned and shelter-housed cats, respectively were vaccinated. Overall, 37.5\% and $48.1 \%$ of cats were vaccinated from Sydney and Perth, respectively. Other categorical data are shown in Table 1. 
Table 1. Descriptive and univariable analysis of risk factors potentially associated with a protective feline parvovirus serum haemagglutination antibody titre $(\geq 1: 40)$ for 523 shelter-housed and owned cats tested from Sydney and Perth.

\begin{tabular}{|c|c|c|c|c|c|c|c|}
\hline \multirow{3}{*}{ Variable } & \multirow{3}{*}{ Category } & \multirow{3}{*}{ Total No. } & \multirow{2}{*}{\multicolumn{2}{|c|}{$\begin{array}{c}\text { Protective FPV } \\
\text { Titre }\end{array}$}} & \multirow{3}{*}{$95 \% \mathrm{CI}$} & \multirow{3}{*}{$X^{2}$-Value } & \multirow{3}{*}{$p$-Value $^{1}$} \\
\hline & & & & & & & \\
\hline & & & No. & $\%$ & & & \\
\hline \multirow{4}{*}{ Group } & Perth owned & 117 & 112 & 95.7 & $90.4-96.8$ & \multirow{4}{*}{11.733} & \multirow{4}{*}{0.008} \\
\hline & Perth shelter & 124 & 115 & 92.7 & $86.7-96.6$ & & \\
\hline & Sydney owned & 172 & 156 & 90.7 & $90.9-96.7$ & & \\
\hline & Sydney shelter & 110 & 110 & 100 & $96.7-100$ & & \\
\hline \multirow{2}{*}{ Shelter vs. Owned } & Shelter & 234 & 225 & 96. & $92.8-98.2$ & \multirow{2}{*}{2.798} & \multirow{2}{*}{0.094} \\
\hline & Owned & 289 & 268 & 92.7 & $89.1-95.5$ & & \\
\hline \multirow{2}{*}{ Location } & Perth & 241 & 227 & 94.2 & $90.4-96.8$ & \multirow{2}{*}{0.004} & \multirow{2}{*}{0.947} \\
\hline & Sydney & 282 & 266 & 94.3 & $90.9-96.7$ & & \\
\hline \multirow{3}{*}{ Age } & $<1$ year & 205 & 188 & 91.7 & $87.05-95.1$ & \multirow{3}{*}{4.154} & \multirow{3}{*}{0.125} \\
\hline & $1-8$ years & 232 & 222 & 95.7 & $92.2-97.9$ & & \\
\hline & $>8$ years & 86 & 83 & 96.5 & $90.1-99.3$ & & \\
\hline \multirow{2}{*}{ Sex } & Male & 241 & 224 & 93 & $89-95.8$ & \multirow{2}{*}{2.202} & \multirow{2}{*}{0.138} \\
\hline & Female & 250 & 240 & 96 & $92.8-98.1$ & & \\
\hline & Desexed & 275 & 263 & 95.6 & $92.5-97.7$ & & \\
\hline Desexing Status & Intact & 195 & 183 & 93.9 & $89.5-96.8$ & 0.755 & 0.686 \\
\hline & Unknown & 19 & 18 & 94.7 & $74.0-99.9$ & & \\
\hline & Domestic & 418 & 403 & 96.4 & $94.2-98.0$ & & \\
\hline Breed & Non-domestic & 53 & 50 & 94.3 & 84.4-98.9 & 0.087 & 0.087 \\
\hline Outdoor A ccesc & Indoors only & 99 & 91 & 91.9 & $84.7-96.5$ & 0788 & 0375 \\
\hline utuor Access & Outdoor access & 253 & 239 & 94.5 & $90.9-96.9$ & 0.700 & 0.373 \\
\hline Dogs in the House & No & 97 & 96 & 99.0 & $94.4-100$ & & \\
\hline Dogs in the House & Yes & 50 & 50 & 100 & 92.9-100 & 0.519 & 0.471 \\
\hline & Breeder/Pet shop & 51 & 48 & 94.1 & 83.8-98.8 & & \\
\hline Source & Shelter/Stray & 275 & 259 & 94.2 & $90.7-96.6$ & 4.435 & 0.109 \\
\hline & Other & 108 & 107 & 99.1 & $95.0-100$ & & \\
\hline & Healthy & 417 & 396 & 95.0 & $92.4-96.9$ & 0085 & 0770 \\
\hline Health Status & Sick/Injured & 71 & 68 & 95.8 & 88.1-99.1 & 0.085 & $0.7 \%$ \\
\hline Vaccination Status & Vaccinated & 218 & 217 & 99.5 & $97.5-100$ & 17617 & $<0.001$ \\
\hline vactiltationt Status & $\begin{array}{l}\text { Unvaccinated or } \\
\text { Unknown }\end{array}$ & 305 & 276 & 90.5 & $86.6-93.5$ & & \\
\hline & MLV only & 114 & 113 & 99.1 & $95.2-100$ & & \\
\hline Vaccination Type & Inactivated only & 18 & 18 & 100 & 81.5-100 & 0.336 & 0.846 \\
\hline & Both & 20 & 20 & 100 & $83.2-100$ & & \\
\hline & $<6$ months & 22 & 21 & 95.5 & 77.16-99.9 & & \\
\hline & 6-12 months & 126 & 126 & 100 & 97.1-100 & & \\
\hline Time Since Last & $1-3$ years & 38 & 38 & 100 & $90.8-100$ & 1.651 & 0.199 \\
\hline & $>3$ years & 22 & 22 & 100 & $84.6-100$ & & \\
\hline & Unknown & 10 & 10 & 100 & & & \\
\hline Medications & No & 446 & 419 & 94.0 & $91.3-96.0$ & & \\
\hline Viedications & Yes & 77 & 74 & 96.1 & $89.0-99.2$ & 0.565 & 0.452 \\
\hline FeLV Antigen & Negative & 82 & 82 & 100 & $95.6-100$ & $\mathrm{n} / \mathrm{a}$ & $\mathrm{n} / \mathrm{a}$ \\
\hline Status & Positive & 0 & 0 & 100 & $95.6-100$ & $\mathrm{n} / \mathrm{a}$ & n/a \\
\hline FIV Antibody & Negative & 105 & 104 & 99.1 & $94.8-100$ & 0.23 & 0.631 \\
\hline Status & Positive & 24 & 24 & 100 & $85.8-100$ & 0.23 & 0.631 \\
\hline
\end{tabular}

\subsection{Seroprevalence and Variables Associated with a Protective FPV Titre}

Overall, 493 of the 523 cats $(94.3 \%)$ had protective FPV antibody titres. The frequency distribution of FPV HI titre results is shown in Figure 2. There was a significant difference in the magnitude of FPV titres between vaccinated cats and cats that were unvaccinated or of unknown vaccination 
status $(p<0.001)$. Among seropositive cats, the median HI titre was 1:160 for unvaccinated/unknown vaccination status cats and 1:640 for vaccinated cats $(p<0.001)$.

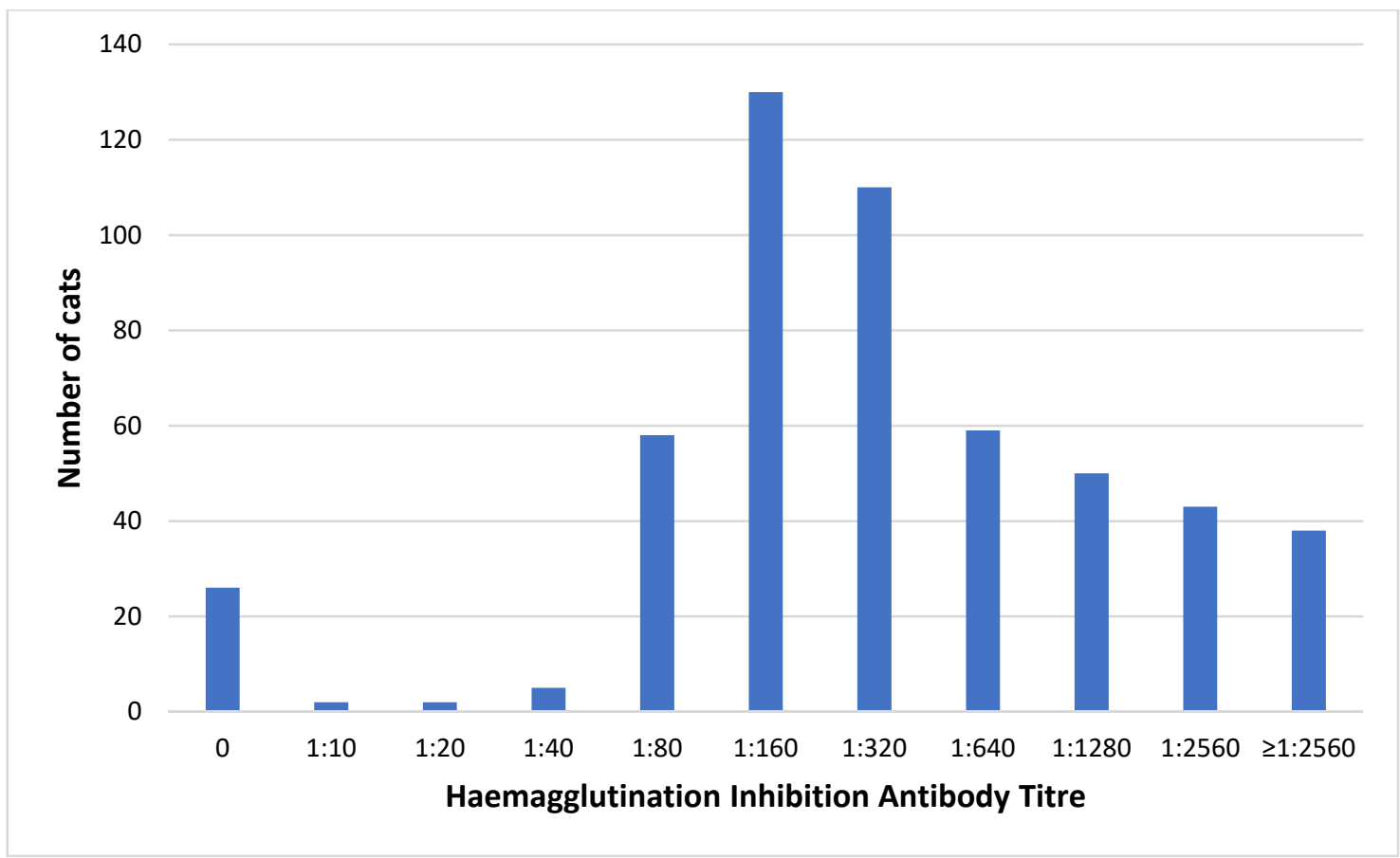

Figure 2. Frequency distribution of serum FPV haemagglutination inhibition titres among all cats tested in this study $(n=523)$.

For the univariable analysis, cats of unknown vaccination status $(n=200)$ and unvaccinated cats $(n=105)$ were considered as one group. There was no significant difference in the presence of FPV protective titres between cats of unknown vaccination status (91\%; CI 86.15\%-94.58\%) and unvaccinated cats $\left(89.5 \%\right.$; CI $82.01 \%-94.65 \%$; $\left.X^{2}=0.1744, p=0.6762\right)$. In the univariable analysis, factors significantly associated with a protective FPV titre were study group, vaccination status (Table 1 ), and three of the four SEIFAs (IRSD $p=0.001$; IRSAD $p=0.001$; IEO $p=0.002$ ).

A multivariable logistic regression analysis was performed to predict FPV protection status. Using a stepwise logistic regression model fit to the significant variables in the univariable analysis, group, and vaccination status, a large standard error for the Sydney shelter group indicated that the variable "group" was problematic to include in this model. A more stable model was achieved using the variables of ownership (owned, shelter-housed) and vaccination status (vaccinated vs. unvaccinated/unowned). The model adequately fit the data (Hosmer-Lemeshow, $p=0.077$ and Nagelkerke $r^{2}$ value $\left.=0.164\right)$. In this model the predictors of having a protective titre were being vaccinated (odds ratio $(\mathrm{OR})=29.94)$ and being a shelter-housed cat $(\mathrm{OR}=2.69)($ Table 2$)$.

Table 2. Results of logistic regression analysis using model of best fit containing the variables: vaccinated, Sydney (outbreak) location, and shelter-housed.

\begin{tabular}{cccccccc}
\hline & B & SE & Wald & df & $p$-Value & OR & 95\% CI \\
\hline Vaccinated & 3.293 & 1.024 & 10.346 & 1 & 0.001 & 29.94 & $3.62-200.4$ \\
Shelter-Housed & 0.988 & 0.416 & 5.635 & 1 & 0.018 & 2.685 & $1.19-6.07$ \\
Constant & 1.140 & 0.407 & 7.843 & 1 & $<0.001$ & 3.128 & - \\
\hline
\end{tabular}

B: beta coefficient; SE: standard error; df: degrees of freedom; OR: odds ratio; CI: confidence interval. 
For logistic regression analysis of SEIFA data, when re-categorised as above median vs. median or below median, the IRSD was the best predictor of protective status and cats from postcodes with above median index of disadvantage (relatively less disadvantaged) were 5.93 times more likely to have protective FPV titres (Table 3). The model adequately fit the data (Hosmer-Lemeshow, $p=0.999$ and Nagelkerke $r^{2}$ value $=0.080$ )

Table 3. Results of logistic regression analysis using model of best fit to analyse the index of relative socio-economic disadvantage (IRSD).

\begin{tabular}{cccccccc}
\hline & B & SE & Wald & df & $p$-Value & OR & 95\% CI \\
\hline IRSD & 1.780 & 0.629 & 7.995 & 1 & 0.005 & 5.928 & $1.73-20.35$ \\
Constant & 2.369 & 0.240 & 97.486 & 1 & 0.000 & 10.684 & -
\end{tabular}

B: beta coefficient; SE: standard error; df: degrees of freedom; OR: odds ratio; CI: confidence interval; IRSD: index of relative social disadvantage.

\section{Discussion}

The prevalence of protective FPV antibody titres was surprisingly high, exceeding 94\% in cats studied. Among vaccinated cats the high prevalence of seroprotection (99.5\%) was not unexpected, since FPV vaccines are highly effective in inducing long-lasting humoral immunity [4]. A similarly high FPV seroprevalence among vaccinated cats (97\%-98\%) was reported in North America in 2004, and in Austria in 2016 [20] (Table 4). However, high seroprevalence among vaccinated cat populations is not universal. For example, an investigation of 350 cats in Germany found that $23 \%$ of cats that had received FPV vaccinations in accordance with current global vaccination guidelines had no detectable serum antibodies $[8,11]$. In that study, in addition to vaccination status, risk factors for being seronegative were comorbid disease and glucocorticoid administration.

Table 4. Seroprevalence of protective serum antibody titres against FPV in domestic cats from different geographic regions.

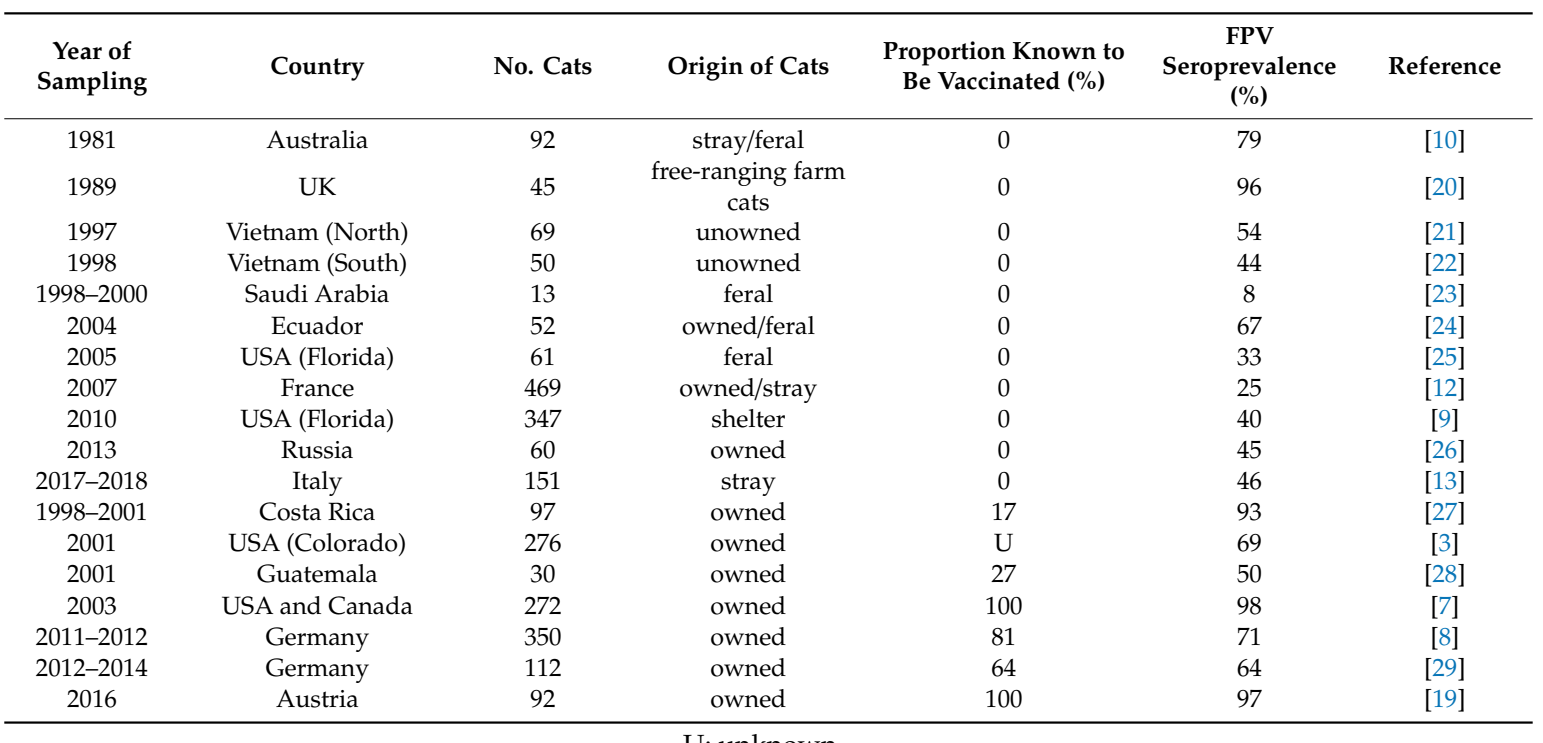

U: unknown.

We identified that a history of FPV vaccination was a strong predictor of protective FPV antibody titres, and the OR of 29.9 among vaccinated cats, was similar to that found in the German study, in which the OR for vaccinated cats having protective FPV titres was 24.8 [8].

The high proportion of seroprotective titres among cats that were unvaccinated or of unknown vaccination status was unexpected. Although it is likely that some cats of unknown vaccination status were in fact vaccinated, there was no significant difference in seroprotection prevalence between 
this group and unvaccinated cats. Serosurveillance of unvaccinated domestic cats in other countries revealed an FPV seroprevalence ranging from $8 \%$ to $96 \%$, although $<50 \%$ of cats were seropositive in 7 of 10 studies [9,12,13,21-27] (Table 4). We used a cut-off HI titre of 1:40 to define seroprotection to allow comparison with the majority of published studies. The WSAVA vaccination guidelines panel considers that the presence of a serum antibody, regardless of titre, is protective [11]. Using that approach would have made little difference to our results, since only five cats had $\mathrm{HI}$ titres above zero but less than 1:40.

Our results indicate that cats in Australia are commonly exposed to Carnivore protoparvovirus 1 in the field. Unvaccinated cats could have had environmental exposure to field or vaccine strains of FPV or CPV. Cats are primarily exposed to FPV through contact with fomites, since this small non-enveloped virus is extremely environmentally resilient and capable of persisting for $>12$ months in favourable conditions [30,31]. Environmental contamination is largely due to faecal shedding of the virus by infected cats, who shed the virus at very high titres for several weeks post-infection. Attenuated vaccine virus strains are also shed in the faeces of vaccinated cats subsequent to replication in the gut $[2,32]$.

The extent to which exposure to CPV in cats provides protection against FPV infection has not been investigated. CPV is antigenically similar to FPV, since these viruses only differ by several amino acid residues in the viral capsid [33]. In experimental studies where cats were inoculated with CPV-2c or CPV-2a orally, all cats developed FPV neutralising $(\mathrm{VN})$ antibody titres, but of a lower magnitude than VN titres to CPV, suggesting that immunity to FPV induced by CPV would be shorter in duration than that induced by FPV [34]. Similarly, sera from cats immunised with attenuated FPV vaccines neutralise $\mathrm{CPV}$, although neutralising antibody titres are two- to eight-fold lower than those induced by $\mathrm{CPV}$, consistent with a shorter duration of immunity [5,35]. Interestingly, seropositive cats that were unvaccinated or of unknown vaccination status in our study had three-fold lower median HI titres than vaccinated cats. This could be due to a number of factors, including exposure to CPV but not FPV, or a decline in FPV titre over time after initial exposure in unvaccinated cats in contrast to vaccinated cats, which may have been re-vaccinated repeatedly over time. In cats that develop FPL, exposure to field strains of parvoviruses by natural infection often induces higher $\mathrm{VN}$ or $\mathrm{HI}$ titres than those induced after vaccination with a homologous strain [34,36]. Whether this is true for cats subclinically infected with FPV is not known. We did not find an association between the presence of dogs in the household and FPV seropositivity, although this is not surprising since exposure to parvoviruses is often indirect. Future investigations to determine CPV- and FPV-specific antibody titres simultaneously are warranted in order to better understand the dynamics of the role of exposure to heterologous parvoviruses in FPV immunity among unvaccinated cats. In addition, or alternatively, the parvoviral strains that cats have been exposed to can be determined by molecular sequencing of persistent episomal parvoviral DNA in blood monocytes post-infection. In two of three studies that used this technique in healthy seropositive cats from Italy and Vietnam, exposure to CPV was found to be as, or more, frequent than to FPV [37-39].

The high rate of FPV seropositivity among cats not known to be vaccinated also indicates that most infections were likely subclinical or caused only mild disease. The development and severity of FPL in naïve cats is dependent on the interplay of multiple host factors (e.g., age, immune status) and viral factors (e.g., inoculating dose), as well as the presence, in some cases, of co-pathogens including intestinal parasites and other enteric viruses (e.g., bocaviruses) [40,41]. Since FPV is profoundly immunosuppressive in cats, opportunistic pathogens, such as bacteria and fungi, can also contribute to the development of severe clinical disease [42,43]. The role of co-pathogens, if any, in the Australian FPL outbreaks, has not been investigated.

Contrary to our expectations, we did not find a significant difference in FPV seroprevalence between outbreak and non-outbreak regions. The high rate of immunity in the outbreak population tested may reflect "boosting" of population immunity among unvaccinated cats due to widespread exposure to FPV in the field, since sera were collected one to two years after the first epizootic outbreaks 
occurred in Sydney. Supporting this, the seroprevalence in the outbreak population was high despite the overall proportion of cats known to be vaccinated (37.5\%) being lower than that of the non-outbreak population $(48 \%)$.

Unlike several other studies in which FPV seropositivity was associated with age $>6$ months or age $>1$ year, reflecting an increasing likelihood of exposure over time, and especially within the first year of life, we did not find an association with age $[9,12,13]$.

The significant difference in seroprevalence among the four groups of cats detected in univariable analysis was likely influenced by the different vaccination proportions among these groups. Owned cats in Sydney were younger than those in Perth because we targeted veterinary clinics in close proximity to the previous FPL outbreaks that were enrolling cats for subsidised desexing programs in the outbreak region.

We could not include group in the multivariable analysis together with vaccination status because of the large standard error (SE) associated with the Sydney shelter group, likely a consequence of the uniform presence of protective titres in this study group. Another potential limitation of our study was that the number of seronegative results was smaller than expected, which might have limited our ability to identify significant predictors of seropositivity in logistic regression analyses. The finding that shelter-housed cats were 2.65 times more likely to have protective FPV titres than owned cats may have been influenced by differences in their exposure history.

Our finding that cats from postcodes of relatively less disadvantage were 5.93 times more likely to have protective FPV titres is useful to inform strategies to achieve more homogeneous immunity to FPV in the cat population, such as the locations and frequency of community companion animal vaccination and pet health educational events provided by veterinary professional organisations and animal charities. The IRSD used in this study is based on measures of wealth and income, including household income, employment status, and level of education obtained from census data [19].

Based on FPV seroprevalences of $68.5 \%$ to $70.6 \%$ among owned populations of cats, it has been suggested that epizootic outbreaks will not occur when the seroprevalence is $>70 \%[11,40]$. However, the critical level of vaccine coverage required to prevent FPL outbreaks is unknown, since the basic reproduction number $\left(R_{0}\right)$ of FPV (i.e., the number of new cases of infection generated by the first infectious individual in a completely susceptible population), has not been modelled for Carnivore protoparvovirus 1 from outbreak data [44]. Simplistically, the critical level of vaccine coverage, or the fraction of the population that is required to be immunised to halt transmission of a pathogen within a population, can be calculated as $1-1 / R_{0}$ [44]. For pathogens with a high $R_{0}$, the critical level of vaccine coverage is very high and outbreaks can occur with relatively small fluctuations in population immunity, even though the vast majority of the population is immune [45]. Disease outbreaks can occur when inhomogeneous vaccine coverage results in susceptible pockets of the population occurring in an otherwise protected population.

While vaccination is undoubtedly the most effective strategy to protect a cat against FPL, administration of FPV vaccines to seropositive cats is unnecessary, ineffective in boosting immunity, and has the potential for adverse effects [46]. FPV antibody titre testing is being increasingly used by veterinarians to determine the timing of vaccine administration in owned cats. Point-of-care (POC) tests with high positive predictive values (PPV) are desirable and enable the decision to vaccinate to be made during the same consultation as testing. POC tests with high PPV are available for FPV titre testing in cats, and since PPV increases with seroprevalence, use of these tests among similar populations of Australian cats to those tested here, would be unlikely to result in seronegative animals not being vaccinated, since the likelihood of incorrect identification of a cat as being seropositive would be very low [6].

Author Contributions: Conceptualisation, V.R.B., E.J. and M.P.W.; methodology, V.R.B., M.P.W., C.D. (Conor Davis), C.B., N.D. and E.J.; project administration, V.R.B. and E.J.; formal analysis, M.P.W.; investigation, E.J., V.R.B., C.D. (Conor Davis), J.A.B., M.C., C.B., N.D., L.B., S.O., M.v.B. and L.B.; resources, V.R.B. and J.A.B.; data curation, E.J.; validation, V.R.B., E.J., C.D. (Conor Davis), C.D. (Costantina Desario) and M.C.; visualisation, E.J. and V.R.B.; 
writing-original draft preparation, E.J.; writing—review and editing, all authors.; supervision, V.B.; funding acquisition, V.B., J.B. and M.W. All authors have read and agreed to the published version of the manuscript.

Funding: This research was funded by the Winn Feline Foundation, grant number W18-006.

Acknowledgments: The authors thank the following people and groups for their assistance in sample collection and survey completion: Roz Robinson, Karen de Souza from Cat Haven, Perth; Kristina Vesk and Nerida Atkin from Cat Protection Society of NSW; Simone Maher, Jaimee-lee Jeffery and Monique Sarkany from the Animal Welfare League NSW; Christine Cole and Bronwyn Evans-Miller, Sydney Cats and Dogs Home; University Veterinary Teaching Hospital Sydney; Abbotsbury Veterinary Clinic; Colyton Veterinary Hospital; Concord Veterinary Hospital; Croydon Park Veterinary Clinic; Greystanes Veterinary Clinic; Leppington Veterinary Clinic; Rooty Hill Veterinary Surgery and Southern Cross Veterinary Clinic. The authors also thank Uwe Truyen for methodological advice.

Conflicts of Interest: The authors declare no conflict of interest. None of the funders had a role in the design of the study; in the analyses, or interpretation of data; in the writing of the manuscript, or in the decision to publish the results.

\section{References}

1. Barrs, V.R. Feline Panleukopenia: A Re-emergent Disease. Vet. Clin. Small Anim. Pract. 2019, 49, 651-670. [CrossRef] [PubMed]

2. Van Brussel, K.; Carrai, M.; Lin, C.; Kelman, M.; Setyo, L.; Aberdein, D.; Brailey, J.; Lawler, M.; Maher, S.; Plaganyi, I.; et al. Distinct Lineages of Feline Parvovirus Associated with Epizootic Outbreaks in Australia, New Zealand and the United Arab Emirates. Viruses 2019, 11, 1155. [CrossRef] [PubMed]

3. Lappin, M.R.; Andrews, J.; Simpson, D.; Jensen, W.A. Use of serologic tests to predict resistance to feline herpesvirus 1, feline calicivirus, and feline parvovirus infection in cats. J. Am. Vet. Med. Assoc. 2002, 220, 38-42. [CrossRef]

4. Scott, F.W.; Geissinger, C.M. Long-term immunity in cats vaccinated with an inactivated trivalent vaccine. Am. J. Vet. Res. 1999, 60, 652-658.

5. Jakel, V.; Cussler, K.; Hanschmann, K.M.; Truyen, U.; Konig, M.; Kamphuis, E.; Duchow, K. Vaccination against Feline Panleukopenia: Implications from a field study in kittens. BMC Vet. Res. 2012, 8, 62. [CrossRef] [PubMed]

6. Mende, K.; Stuetzer, B.; Truyen, U.; Hartmann, K. Evaluation of an in-house dot enzyme-linked immunosorbent assay to detect antibodies against feline panleukopenia virus. J. Feline Med. Surg. 2014, 16, 805-811. [CrossRef]

7. Mouzin, D.E.; Lorenzen, M.J.; Haworth, J.D.; King, V.L. Duration of serologic response to three viral antigens in cats. J. Am. Vet. Med. Assoc. 2004, 224, 61-66. [CrossRef]

8. Mende, K.; Stuetzer, B.; Sauter-Louis, C.; Homeier, T.; Truyen, U.; Hartmann, K. Prevalence of antibodies against feline panleukopenia virus in client-owned cats in Southern Germany. Vet. J. 2014, 199, 419-423. [CrossRef]

9. DiGangi, B.A.; Levy, J.K.; Griffin, B.; McGorray, S.P.; Dubovi, E.J.; Dingman, P.A.; Tucker, S.J. Prevalence of serum antibody titers against feline panleukopenia virus, feline herpesvirus 1 , and feline calicivirus in cats entering a Florida animal shelter. J. Am. Vet. Med. Assoc. 2012, 241, 1320-1325. [CrossRef]

10. Coman, B.J.; Jones, E.H.; Westbury, H.A. Protozoan and viral infections of feral cats. Aust. Vet. J. 1981, 57, 319-323. [CrossRef]

11. Day, M.J.; Horzinek, M.C.; Schultz, R.D.; Squires, R.A. WSAVA Guidelines for the vaccination of dogs and cats. J. Small Anim. Pract. 2010, 51, e1-e32. [CrossRef]

12. Hellard, E.; Fouchet, D.; Santin-Janin, H.; Tarin, B.; Badol, V.; Coupier, C.; Leblanc, G.; Poulet, H.; Pontier, D. When cats' ways of life interact with their viruses: A study in 15 natural populations of owned and unowned cats (Felis silvestris catus). Prev. Vet. Med. 2011, 101, 250-264. [CrossRef]

13. Dall'Ara, P.; Labriola, C.; Sala, E.; Spada, E.; Magistrelli, S.; Lauzi, S. Prevalence of serum antibody titres against feline panleukopenia, herpesvirus and calicivirus infections in stray cats of Milan, Italy. Prev. Vet. Med. 2019, 167, 32-38. [CrossRef]

14. Dhand, N.K.; Khakar, M.S. Statulator: An online statistical calculator. Sample Size Calculator for Comparing Two Independent Proportions. Available online: http://statulator.com/SampleSize/ss2P.html (accessed on 5 January 2020). 
15. Select Statistical Services Odds Ratio-Sample Size. Available online: https://select-statistics.co.uk/calculators/ sample-size-calculator-odds-ratio/ (accessed on 16 March 2020).

16. Cavalli, A.; Desario, C.; Marinaro, M.; Losurdo, M.; Camero, M.; Decaro, N.; Catella, C.; Lanave, G.; Buonavoglia, C. Oral administration of modified live canine parvovirus type $2 \mathrm{~b}$ induces systemic immune response. Vaccine 2020, 38, 115-118. [CrossRef]

17. Johnson, R.H. Serologic procedures for the study of feline panleukopenia. J. Am. Vet. Med. Assoc. 1971, 158, 876.

18. Duijvestijn, M.; Mughini-Gras, L.; Schuurman, N.; Schijf, W.; Wagenaar, J.A.; Egberink, H. Enteropathogen infections in canine puppies: (Co-)occurrence, clinical relevance and risk factors. Vet. Microbiol. 2016, 195, 115-122. [CrossRef]

19. Australian Bureau of Statistics. Postal Area, SA1 Distributions, SEIFA 2011; Australian Bureau of Statistics: Canberra, Australia, 2013.

20. Haselberger, A.; Tichy, A.; Mostl, K. Evaluation of antibody titres against feline Panleukopenia Virus, feline Herpesvirus-1 and feline Calicivirus in cats in eastern Austria. Wien. Tierarztl. Mon. 2016, 103, 149-161.

21. Yamaguchi, N.; Macdonald, D.W.; Passanisi, W.C.; Harbour, D.A.; Hopper, C.D. Parasite prevalence in free-ranging farm cats, Felis silvestris catus. Epidemiol. Infect. 1996, 116, 217-223. [CrossRef]

22. Miyazawa, T.; Ikeda, Y.; Nakamura, K.; Naito, R.; Mochizuki, M.; Tohya, Y.; Vu, D.; Mikami, T.; Takahashi, E. Isolation of feline parvovirus from peripheral blood mononuclear cells of cats in northern Vietnam. Microbiol. Immunol. 1999, 43, 609-612. [CrossRef]

23. Nakamura, K.; Ikeda, Y.; Miyazawa, T.; Nguyen, N.T.P.; Duong, D.D.; Le, K.H.; Vo, S.D.; Phan, L.V.; Mikami, T.; Takahashi, E. Comparison of prevalence of feline herpesvirus type 1, calicivirus and parvovirus infections in domestic and leopard cats in Vietnam. J. Vet. Med Sci. 1999, 61, 1313-1315. [CrossRef]

24. Ostrowski, S.; Van Vuuren, M.; Lenain, D.M.; Durand, A. A serologic survey of wild felids from central west Saudi Arabia. J. Wildl. Dis. 2003, 39, 696-701. [CrossRef]

25. Levy, J.; Crawford, P.; Lappin, M.; Dubovi, E.; Levy, M.; Alleman, R.; Tucker, S.; Clifford, E. Infectious diseases of dogs and cats on Isabela Island, Galapagos. J. Vet. Intern. Med. 2008, 22, 60-65. [CrossRef]

26. Fischer, S.M.; Quest, C.M.; Dubovi, E.J.; Davis, R.D.; Tucker, S.J.; Friary, J.A.; Crawford, P.C.; Ricke, T.A.; Levy, J.K. Response of feral cats to vaccination at the time of neutering. J. Am. Vet. Med. Assoc. 2007, 230, 52-58. [CrossRef]

27. Pavlova, E.V.; Kirilyuk, V.E.; Naidenko, S.V. Patterns of seroprevalence of feline viruses among domestic cats (Felis catus) and Pallas' cats (Otocolobus manul) in Daursky Reserve, Russia. Can. J. Zool. 2015, 93, 849-855. [CrossRef]

28. Blanco, K.; Prendas, J.; Cortes, R.; Jimenez, C.; Dolz, G. Seroprevalence of Viral Infections in Domestic Cats in Costa Rica. J. Vet. Med Sci. 2009, 71, 661-663. [CrossRef]

29. Lickey, A.L.A.; Kennedy, M.; Patton, S.; Ramsay, E.C. Serologic survey of domestic felids in the Peten region of Guatemala. J. Zool. Wildl. Med. 2005, 36, 121-123. [CrossRef]

30. Johnson, R.H. Feline panleucopaenia. Vet. Rec. 1969, 84, 338-340. [CrossRef]

31. Johnson, R.H. Feline panleucopaenia virus. 3. Some properties compared to a feline herpes virus. Res. Vet. Sci. 1966, 7, 112-115. [CrossRef]

32. Bergmann, M.; Schwertler, S.; Speck, S.; Truyen, U.; Reese, S.; Hartmann, K. Faecal shedding of parvovirus deoxyribonucleic acid following modified live feline panleucopenia virus vaccination in healthy cats. Vet. Rec. 2019, 185, 83. [CrossRef]

33. Hoelzer, K.; Parrish, C.R. The emergence of parvoviruses of carnivores. Vet. Res. 2010, 41, 39. [CrossRef]

34. Nakamura, K.; Ikeda, Y.; Miyazawa, T.; Tohya, Y.; Takahashi, E.; Mochizuki, M. Characterisation of cross-reactivity of virus neutralising antibodies induced by feline panleukopenia virus and canine parvoviruses. Res. Vet. Sci. 2001, 71, 219-222. [CrossRef]

35. Truyen, U.; Parrish, C.R. Feline panleukopenia virus: Its interesting evolution and current problems in immunoprophylaxis against a serious pathogen. Vet. Microbiol. 2013, 165, 29-32. [CrossRef]

36. Jack, S.C.; Sutton, D.; Bhogle, A.; Spibey, N.; Francis, M. FPL-vaccinated cats are protected from CPV2c and CPV2b challenge. Vet. Rec. 2014, 174, 146. [CrossRef]

37. Balboni, A.; Bassi, F.; De Arcangeli, S.; Zobba, R.; Dedola, C.; Alberti, A.; Battilani, M. Molecular analysis of carnivore Protoparvovirus detected in white blood cells of naturally infected cats. BMC Vet. Res. 2018, $14,41$. [CrossRef] 
38. Ikeda, Y.; Mochizuki, M.; Naito, R.; Nakamura, K.; Miyazawa, T.; Mikami, T.; Takahashi, E. Predominance of canine parvovirus (CPV) in unvaccinated cat populations and emergence of new antigenic types of CPVs in cats. Virology 2000, 278, 13-19. [CrossRef]

39. Marenzoni, M.L.; Antognoni, M.T.; Baldelli, F.; Miglio, A.; Stefanetti, V.; Desario, C.; Di Summa, A.; Buonavoglia, C.; Decaro, N. Detection of parvovirus and herpesvirus DNA in the blood of feline and canine blood donors. Vet. Microbiol. 2018, 224, 66-69. [CrossRef]

40. Stuetzer, B.; Hartmann, K. Feline parvovirus infection and associated diseases. Vet. J. 2014, 201, 150-155. [CrossRef]

41. Piewbang, C.; Kasantikul, T.; Pringproa, K.; Techangamsuwan, S. Feline bocavirus-1 associated with outbreaks of hemorrhagic enteritis in household cats: Potential first evidence of a pathological role, viral tropism and natural genetic recombination. Nat. Sci. Rep. 2019, 9, 16367. [CrossRef]

42. Foley, J.E.; Orgad, U.; Hirsh, D.C.; Poland, A.; Pedersen, N.C. Outbreak of fatal salmonellosis in cats following use of a high-titer modified-live panleukopenia virus vaccine. J. Am. Vet. Med. Assoc. 1999, 214, 67-70.

43. Ossent, P. Systemic aspergillosis and mucormycosis in 23 cats. Vet. Rec. 1987, 120, 330-333. [CrossRef]

44. Metcalf, C.J.E.; Ferrari, M.; Graham, A.L.; Grenfell, B.T. Understanding Herd Immunity. Trends Immunol. 2015, 36, 753-755. [CrossRef]

45. Guerra, F.M.; Bolotin, S.; Lim, G.; Heffernan, J.; Deeks, S.L.; Li, Y.; Crowcroft, N.S. The basic reproduction number (R0) of measles: A systematic review. Lancet Infect. Dis. 2017, 17, e420-e428. [CrossRef]

46. Bergmann, M.; Schwertler, S.; Reese, S.; Speck, S.; Truyen, U.; Hartmann, K. Antibody response to feline panleukopenia virus vaccination in healthy adult cats. J. Feline Med. Surg. 2018, 20, 1087-1093. [CrossRef]

(C) 2020 by the authors. Licensee MDPI, Basel, Switzerland. This article is an open access article distributed under the terms and conditions of the Creative Commons Attribution (CC BY) license (http://creativecommons.org/licenses/by/4.0/). 